\title{
Biodiversity, Habitat Area, Resource Growth Rate and Interference Competition
}

\author{
YANG KUANG* \\ Department of Mathematics and Statistics, \\ Arizona State University, \\ Tempe, AZ 85287-1804, \\ U.S.A. \\ E-mail:kuang@asu.edu \\ WILLIAM F. FAGAN \\ Department of Biology, \\ University of Maryland, \\ College Park, MD 20742, \\ U.S.A. \\ E-mail: bfagan@glue.umd.edu \\ IRAKLI LOLADZE \\ Department of Ecology and Evolutionary Biology, \\ Princeton University, \\ Princeton, NJ 08544-1033, \\ U.S.A. \\ E-mail: iloladze@princeton.edu.
}

For the majority of species, per capita growth rate correlates negatively with population density. Although the popular logistic equation for the growth of a single species incorporates this intraspecific competition, multi-trophic models often ignore self-limitation of the consumers. Instead, these models often assume that the predator-prey interactions are purely exploitative, employing simple Lotka-Volterra forms in which consumer species lack intraspecific competition terms. Here we show that intraspecific interference competition can account for the stable coexistence of many consumer species on a single resource in a homogeneous environment. In addition, our work suggests a potential mechanism for field observations demonstrating that habitat area and resource productivity strongly positively correlate to biodiversity. In the special case of a modified Lotka-Volterra model describing multiple predators competing for a single resource, we present an ordering procedure that determines the deterministic fate of each specific consumer. Moreover, we find that the growth rate of a resource species is proportional to the maximum number of consumer species that resource can support. In the limiting case, when the resource growth rate is infinite, a model with intraspecific interference reduces to the conventional Lotka-Volterra competition model where there can be an unlimited number of coexisting consumers.

\footnotetext{
*Author to whom correspondence should be addressed.
} 
This highlights the crucial role that resource growth rates may play in promoting coexistence of consumer species.

(C) 2003 Society for Mathematical Biology. Published by Elsevier Science Ltd. All rights reserved.

\section{INTRODUCTION}

Many previous modeling efforts led to the development of the so-called competitive exclusion principle (CEP), one of the oldest and most intriguing paradigms in community ecology. The CEP states that at most $n$ species can coexist on $n$ resources (Volterra, 1926; Hardin, 1960; MacArthur and Levins, 1964; Levin, 1970; Abrams, 1980; Grover, 1997). These coexistence results stem from the mathematically convenient assumption that consumers neither interact nor interfere with each other directly. Although mathematically rigorous, the above formulation of the CEP fails to answer the basic question of the existence of biodiversity.

Advances in theoretical ecology have identified various mechanisms that promote coexistence, demonstrating that the above formulation of the CEP is too restrictive. Modified CEPs have been proposed (Levin, 1970; Armstrong and McGehee, 1980; Loladze et al., 2003) that make different assumptions about the nature of consumer limitation. It is also well known that for models that explicitly consider resources, the coexistence of $n$ species on fewer than $n$ resources is possible via internally produced limit cycles or chaotic fluctuations (Armstrong and McGehee, 1976, 1980; Huisman and Weissing, 1999). Other factors such as spatial heterogeneity (Tilman, 1994; Neuhauser, 1998; Kinzig et al., 1999) or temporal heterogeneity (Hsu et al., 1981), chaotic advection in aquatic systems (Karolyi et al., 2000), disturbance (Hastings, 1980; Abrams, 1984a), direct interference between consumers given constant or consumer-dependent resource input rates (Vance, 1984, 1985), and stoichiometric principles (Loladze et al., 2003) provide mechanisms that enhance coexistence. Although these mechanisms may promote biodiversity, mathematical efforts thus far suggest that they can typically only add one or just a few more coexisting consumer species for each explicitly modeled resource species (Hsu et al., 1981; Li and Smith, 2002). Computational efforts show that many more species may coexist in a fluctuating environment setting with at least three resources (Armstrong and McGehee, 1976; Huisman and Weissing, 1999, $2000,2001)$. Other relevant work deals with the impact of food web connectivity on the biodiversity and stability of ecosystems (McCann et al., 1998; McCann, 2000). Despite these advances, our knowledge still falls short of explaining the vast biodiversity we witness in Nature (Hutchinson, 1961; Levin, 1999).

As May (2001, pp. 80-81) points out the logistic, Gompertz and other equations that theoretically capture self-limitation of a resource have stabilizing effects on consumer-resource interactions. Moreover, May cites Tanner's (1966) review that provides empirical support for negative density dependence: of 71 species for 
which adequate data were available, 46 had a significant negative correlation between population density and per capita growth rate, 15 had negative but not significant correlations, seven had no correlations, whereas only one (humans) had a significant positive correlation. A recent study (Myers et al., 1995) shows that direct intraspecific competition or intraspecific interference involving negative density dependence appears prevalent in fish populations. Moreover, a comparison of various predator-prey models with experimental data suggests that incorporating intraspecific competition dramatically improves model fits to some well documented experimental data (Harrison, 1995).

Within a population of consumers, intraspecific interference can involve a variety of behavioral mechanisms. Ranked in order of increasing intensity, these can include aggressive displays and posturing, fighting, and intraspecific killing (e.g., infanticide) and cannibalism (Fox, 1975; Krebs and Davies, 1981). When the principal effect of the intraspecific interference is a reduced rate of feeding or resource intake, the effects are probably best modeled via an altered functional response for the consumer (DeAngelis et al., 1975; Cosner et al., 1999; Skalski and Gilliam, 2001). However, even adaptive foraging can lead to intraspecific interference (Abrams, 1984b). In contrast, when lethal fighting or cannibalism occurs, it is more appropriate to build a model in which the dynamics include an additional mortality or loss term for the consumer. Even if the effect is a gradual one, such as when premature death results from wounding or the devotion of substantial energetic resources to aggressive behaviors, it may be appropriate to include a mortality or loss term to capture the wastage effect. The effects of such lethal intraspecific interference will likely be positively density dependent, reflecting the greater potential for intraspecific aggression as individuals become more crowded (Fig. 1). Furthermore, although we do not discuss it in depth here, direct interference competition resulting in mortality can also occur between species. Durant (1998) documents cases of interspecific interference among African carnivores, specifically the killing of cheetah cubs by lions and hyaenas. Tannerfeldt et al. (2002) discuss similar dynamics between fox species.

Although the popular logistic growth equation $\left(x^{\prime}(t) / x(t)=r(1-x(t) / K)\right)$ for single species incorporates this intraspecific competition, it is often overlooked in literature when modeling species at higher trophic levels, where such competition may also be prevalent. Specifically, most mathematical modelers, due to the lack of experimental data and the desire to make models tractable, hastily assume that competition within consumer species is purely exploitative, ignoring contributions of direct or indirect interference. Exceptions to this include a chemostat-type competition model with nutrient recycling in Ruan and He (1998), a general model of two predators competing for a prey by Farkas and Freedman (1989), and a predator-prey model by Cavani and Farkas (1994).

In this paper, we show that intraspecific competition modeled as direct interference at the consumer level can provide for the stable coexistence of many species on few resources. In the special case of Lotka-Volterra type interactions, 

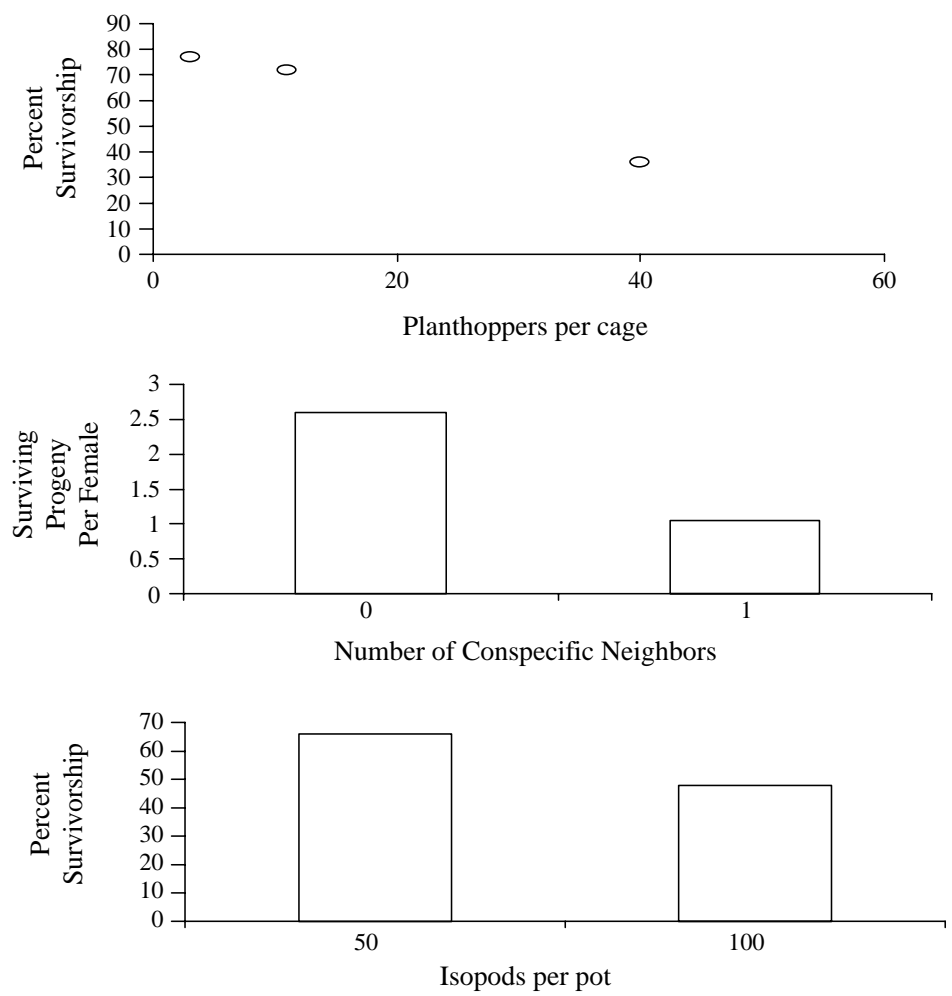

Figure 1. Direct interference resulting in mortality for three species of consumers. In the top figure, survivorship of planthoppers decreases with increasing density due to the effects of intraspecific interference mediated indirectly via plant tissue quality [redrawn from Denno and Roderick (1992)]. In the middle figure, the adults of the whitefly parasitioid Encarsia sophia engage in 'host feeding' [redrawn from Jervis and Kidd (1986)], killing conspecific juveniles when they attack previously parasitized whitefly hosts (Collier and Hunter, 2001). In the bottom figure, direct interference within a population of soil isopods occurs via cannibalism, which increases with increasing density [redrawn from Grosholz (1992)].

this conclusion is mathematically well known and is reported in many important references [Case and Gilpin (1974), Goh (1977), Case and Casten (1979), Abrams (1984b, 1986, 1987, 1998, 2001); all these references argued that intraspecific interference is common]. Although it is argued that spatial heterogeneity and dispersion can mediate the coexistence of an unlimited number of consumers on as few as a single resource (Tilman, 1994), intraspecific interference competition provides a powerful mechanism for species coexistence in more spatially homogeneous situations. We also confirm that resource species growth rates play an important role in sustaining such coexistence. In the special case of modified Lotka-Volterra models, we find that the resource growth rate is almost proportional to the maximum number of consumer species supportable by a single resource. This novel result suggests that resource growth rates may play even greater roles 
than habitat area in promoting coexistence of consumer species. We note here that our result is complementary to the work of Lehman and Tilman (2000) where the authors show through several models that greater diversity increases the temporal stability of the entire community and increases community productivity.

\section{The MODEL AND ITS IMPLICATIONS}

We consider the simple scenario with $n$ consumer (or predator) species $y_{i}, i=$ $1, \ldots, n,\left(y_{i}\right.$ represents the total biomass of $i$ th consumer species in the model below) competing for a single limited resource (or prey) $x$ (which stands for the total resource biomass in the model below) in a closed and homogeneous environment. The following model is a generalization of the Gause-type predatorprey model (Freedman, 1980),

$$
\begin{cases}x^{\prime}(t)=x g(x)-\sum_{i=1}^{n} y_{i} p_{i}(x), & K>x(0)>0, \\ y_{i}^{\prime}(t)=y_{i}\left(c_{i} p_{i}(x)-d_{i}-m_{i} y_{i}-\sum_{j \neq i}^{n} m_{i j} y_{j}\right), & y_{i}(0)>0\end{cases}
$$

$i=1,2, \ldots, n$. All parameters in (2.1) are nonnegative. Here $g(x)$ is the resource per capita growth rate in the absence of predators. It often takes the form of $1-x / K$, where $K$ is the carrying capacity. The predator's functional response $p_{i}(x)$ often takes the form of $p_{i} x$, or $s_{i} x /\left(1+a_{i} x\right) . c_{i}$ is the conversion efficiency of consumer $y_{i}$, and $d_{i}$ represents consumer $y_{i}$ 's constant death rate. $m_{i}>0$ measures the consumer $y_{i}$ 's direct interference with each other, or the so-called intraspecific competition strength that induces additional per capita mortality or loss rate $m_{i} y_{i}$. We may interpret $m_{i}$ not only as mortality but more generally as a loss term. For example, aggressive behavior leads to biomass loss due to higher respiration even if no increased mortality follows. It is the presence of this term that reflects the negative correlation between density and per capita growth rate observed in empirical studies.

In general, direct interspecific competition may also be present. To incorporate such competition, we assume that the direct interspecific effect from consumer species $y_{j}$ inflicts an additional death or loss rate on consumer species $y_{i}$ by approximately $m_{i j} y_{j}$. For example, stoichiometric models (Loladze et al., 2000, 2003) with linear functional responses yield both intraspecific and interspecific competition terms exactly as in model (2.1) due to indirect competition of consumers for some limiting nutrient. It is often observed that consumers interfere with potential competitors from other species. This effect is especially true when the competitors are predatory and different in size. When interference becomes lethal such a scenario connects strongly with the ideas of 'intra-guild predation' 
and 'size-dependent predation', both of which are heavily referenced in ecological journals [e.g., Holt and Polis (1997), Tannerfeldt et al. (2002)].

For mathematical simplicity and convenience, we assume below that

(A1): Functions $g(x)$ and $p_{i}(x), i=1, \ldots, n$ are continuously differentiable, and $p_{i}(x)>0, p^{\prime}(x)>0$ for $x>0$.

From standard differential equation theory (Hale, 1980), the solution of (2.1) is unique and will stay positive for $t>0$. To conform to the general form of single species growth dynamics, we also assume that

(A2): There is a positive constant $K$ (the carrying capacity of the resource species) such that $g(x)>0$ for $x \in[0, K)$ and $g(x)<0$ for $x>K$.

It is easy to see that if $n \geq 2$ and $m_{i}=0, m_{i j}=0, i, j=1, \ldots, n$, then model (2.1) cannot possess any positive steady state, since it is almost impossible that any positive constant, $x^{*}$, satisfies $c_{i} p_{i}(x)-d_{i}=0, i=1, \ldots, n$ simultaneously. When the parameters $c_{i}$ and $d_{i}$ are chosen at random, then with probability one, no two or more of these conditions are simultaneously satisfied. This implies that at most one consumer species can persist. This is the essence of the CEP when there is only one resource and no consumer intraspecific competitions.

In the following, we denote by $E^{*}=\left(x^{*}, y_{1}^{*}, \ldots, y_{n}^{*}\right)$ a possible steady state of model (2.1).

In the limiting case, when the resource growth rate is infinite, one can regard the resource level as staying at the equilibrium level, i.e., $x(t) \equiv x^{*}$. In such a case, the model (2.1) reduces to the conventional Lotka-Volterra competition model, where any number of consumer species can coexist (Strobeck, 1973). Such a scenario occurs when the intraspecific competition force (measured by the intraspecific coefficient) of each species is larger than the sum of all its interspecific competition forces, i.e., $m_{i}>\sum_{j \neq i}^{n} m_{i j}$. Thus, our theoretical model provides a bridge to popular, but highly artificial, competition models that do not explicitly model resource dynamics. In short, high growth rate promotes biodiversity and the idealized infinite resource growth rate results in a competition model that enables the coexistence of unlimited competing species.

Let $m_{i i}=m_{i}, i=1, \ldots, n$. For convenience, we define $M=\left(m_{i j}\right)_{n \times n}$, $y^{*}=\left(y_{1}^{*}, y_{2}^{*}, \ldots, y_{n}^{*}\right)$, and

$$
\begin{aligned}
& p(x)=\left(p_{1}(x), p_{2}(x), \ldots, p_{n}(x)\right) \\
& q(x)=\left(c_{1} p_{1}(x)-d_{1}, c_{2} p_{2}(x)-d_{2}, \ldots, c_{n} p_{n}(x)-d_{n}\right) .
\end{aligned}
$$

Assume that $M$ is invertible. Then

$$
x^{*} g\left(x^{*}\right)=\sum_{i=1}^{n} M^{-1} q\left(x^{*}\right) p\left(x^{*}\right),
$$


and

$$
y^{*}=M^{-1} q\left(x^{*}\right) .
$$

If $m_{i j}=0, i \neq j$, then

$$
x^{*} g\left(x^{*}\right)=\sum_{i=1}^{n} \frac{1}{m_{i}} p_{i}\left(x^{*}\right)\left(c_{i} p_{i}\left(x^{*}\right)-d_{i}\right),
$$

and

$$
y_{i}^{*}=\left(c_{i} p_{i}\left(x^{*}\right)-d_{i}\right) / m_{i} .
$$

If $x^{*}>0$ and $y_{i}^{*}>0, i=1,2, \ldots, n$, then $E^{*}$ is a positive steady state. Let $x_{i}^{b}$ be the 'break-even' prey biomass for predator $y_{i}$, where the birth rate and densityindependent death rate are equal. Specifically,

$$
c_{i} p_{i}\left(x_{i}^{b}\right)=d_{i} .
$$

With $x(0) \in(0, K)$, a standard comparison argument shows that $x(t) \in(0, K)$ for all $t>0$. Mathematically, even if $x(0) \geq K$, it can be shown that $x(t) \in(0, K)$ for large $t>0$ as long as at least one predator's break-even prey biomass is less than the carrying capacity. It is also easy to see that if $x_{i}^{b} \geq K$, then $y_{i}^{\prime}(t)<0$ and hence predator $y_{i}$ will die out. This is equivalent to:

Proposition 2.1. If a predator's break-even prey biomass is greater than the carrying capacity of the resource species, then the predator will go extinct.

A straightforward implication of this proposition is that if we increase the carrying capacity $K$ (which is often referred to measure the size or quality of the habitat), then more species will have their break-even prey biomasses fall below $K$. This enhances the likelihood that some of them will persist. In short, an increase in the resource carrying capacity $K$ promotes biodiversity at the consumer level.

In light of the above proposition, we assume in the rest of this paper that all predators' break-even prey biomasses are less than the carrying capacity of the resource species. Let

$$
x_{M}=\max \left\{x_{i}^{b} \mid i=1,2, \ldots, n\right\}, \quad x_{m}=\min \left\{x_{i}^{b} \mid i=1,2, \ldots, n\right\} .
$$

Then it can be shown that $x_{m} \leq x^{*} \leq x_{M}$.

In the following theorem, we present a simple sufficient condition for $E^{*}$ to be locally attractive if it exists and is positive (i.e., all its components are positive). The condition (2.5) roughly means that the weighted intraspecific competition effect of each consumer species dominates half of the weighted total interspecific competition effects to and from other consumer species [when $p(x)=p x$, (2.5) becomes $\left.2 m_{i}>\sum_{j \neq i}^{n} m_{i j}+\sum_{j \neq i}^{n}\left(c_{i} / c_{j}\right) m_{j i}\right]$. This condition is easily satisfied if interspecific competition effects are weak in comparison to intraspecific competition effects. 
Theorem 2.1. Assume that (2.1) has a positive steady state $E^{*}, m_{i j} \geq 0$, and

$$
\frac{p_{i}\left(x^{*}\right)}{c_{i} p_{i}^{*}}\left(2 m_{i}-\sum_{j \neq i}^{n} m_{i j}\right)-\sum_{j \neq i}^{n} \frac{m_{j i} p_{j}\left(x^{*}\right)}{c_{j} p_{j}^{*}}>0 .
$$

Assume further that

$$
x^{*} g^{\prime}\left(x^{*}\right)+g\left(x^{*}\right)-\sum_{i=1}^{n} y_{i}^{*} p_{i}^{\prime}\left(x^{*}\right) \equiv-\alpha<0 .
$$

Then $E^{*}$ is locally attractive (i.e., all positive solutions near $E^{*}$ tend to $E^{*}$ ).

The following are some important and direct biological implications of Theorem 2.1.

1. If there is no direct interspecific competition among consumer species, then condition (2.5) holds naturally. In this case, if $\left.\frac{d(x g(x))}{d x}\right|_{x=x^{*}}<0$, then from equation (2.6), it follows that $E^{*}$ is stable if it is positive. Notice that $\left.\frac{d(x g(x))}{d x}\right|_{x=x^{*}}<0$ means that the resource species endures self-limitation at its equilibrium biomass. This is equivalent to the simple geometric criterion: if at $x^{*}$, the net growth rate function $(x g(x))$ of the prey has a negative slope, then $E^{*}$ is locally stable. If $x g(x)$ has only one hump, then $E^{*}$ is stable if $x^{*}$ is located at the right-hand side of the hump. It is easy to see that $E^{*}$ remains stable even if $x^{*}$ is slightly to the left-hand side of this hump (Rosenzweig and MacArthur, 1963).

2. The above theorem, equations (2.3) and (2.6) suggest that intraspecific competition of consumers makes positive multi-species equilibrium feasible, while intraspecific competition of the resource promotes the stability of such an equilibrium. Hence, stabilizing properties of intraspecific limitation can provide a simple explanation to the fact that the majority of populations in nature do not oscillate persistently (Krukonis and Schaffer, 1991; Kendall et al., 1998, 1999).

Figure 2 illustrates a solution of model (2.1) with $n=4$, which clearly tends to a stable positive steady state $E^{*}$.

The general qualitative dynamics of model (2.1) are complicated even when there is only one consumer. In this case the model reduces to

$$
\begin{cases}x^{\prime}(t)=x g(x)-y p(x), & x(0)>0, \\ y^{\prime}(t)=y(c p(x)-d-m y), & y(0)>0 .\end{cases}
$$

If the prey grows logistically and the predator exhibits a Holling type II functional response such that $g(x)=1-x / K$ and $p(x)=s x /(1+a x)$, then (2.7) becomes

$$
\begin{cases}x^{\prime}(t)=x(1-x / K)-y s x /(1+a x), & x(0)>0, \\ y^{\prime}(t)=y(\operatorname{cs} x /(1+a x)-d-m y), & y(0)>0 .\end{cases}
$$




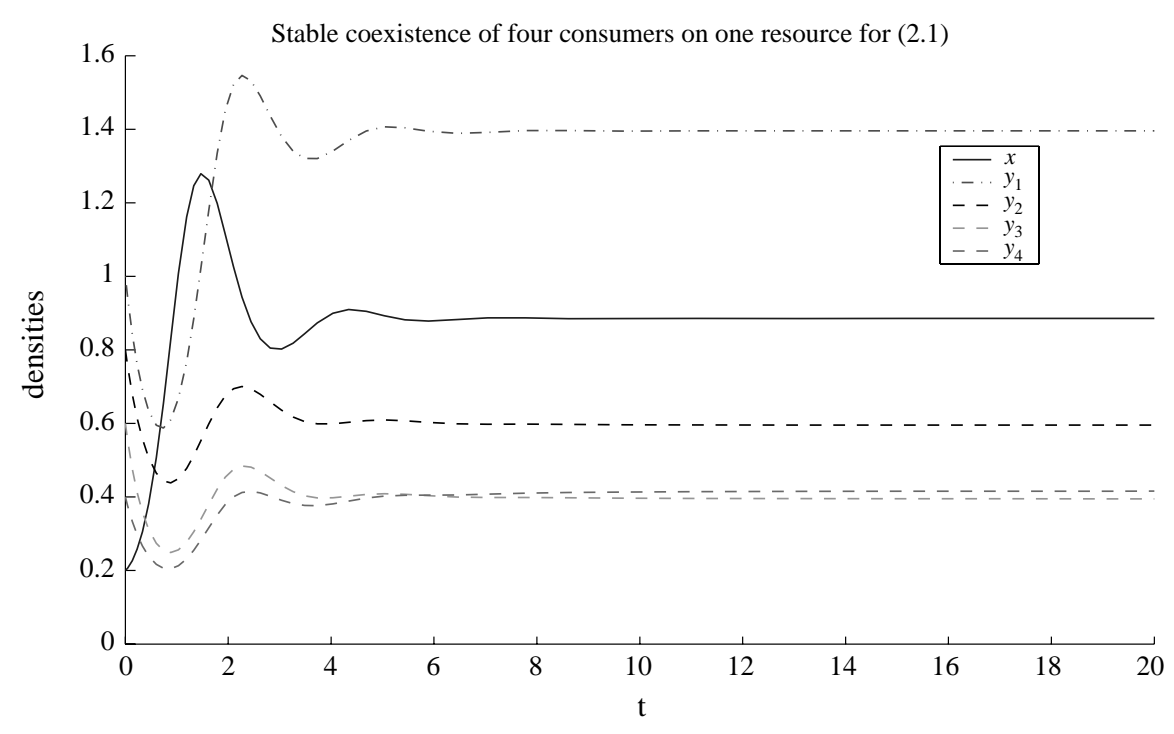

Figure 2. A solution of (2.1) with $g(x)=1-x / K$ and $p_{i}(x)=p_{i} x /\left(1+a_{i} x\right)$, $i=1,2, \ldots, n$. Here $n=4, r=5, K=2.5, p_{1}=1, p_{2}=2, p_{3}=1.5, p_{4}=1.8$, $d_{1}=2, d_{2}=1, d_{3}=1.8, d_{4}=1.5, m_{1}=0.71, m_{2}=0.81, m_{3}=0.91, m_{4}=1$, $c_{1}=3, c_{2}=1, c_{3}=2, c_{4}=1.5, a_{1}=0.24, a_{2}=0.22, a_{3}=0.26, a_{4}=0.28$ and initial condition $(0.2,1,0.8,0.6,0.4)$. This solution tends to a stable positive steady state.

System (2.8) is called the Bazykin model (Bazykin et al., 1998; Kuznetsov, 1998, p. 325). Short of chaotic dynamics, the Bazykin model is capable of generating very complicated and intriguing dynamics. For example, it may have three positive steady states and several limit cycles, and it may undergo several codimension 2 bifurcations and generate a large homoclinic loop. Indeed, many mathematical questions on the qualitative properties of the model remain open (Hwang and Kuang, 2002). An in-depth mathematical treatment of the full model will undoubtedly require much more technical effort and will be pursued separately. If $m=0$ in (2.8), then it is the well studied conventional Holling type II predator-prey model (Kuang, 1990). For this technical reason, we study in detail in the next section the simple case of (2.1) when $p_{i}(x)$ takes the form of $p_{i} x$ and $g(x)=r(1-x / K)$. For this special Lotka-Volterra situation, we present an almost complete global qualitative analysis and point out some important biological implications. More general and in-depth comments on model (2.1) will be given in the discussion section.

\section{LOTKA-VOLTERRA TYPE MODEL AND ITS IMPLICATIONS}

In this section, we assume that in (2.1), $g(x)=r(1-x / K)$ and $p_{i}(x)=p_{i} x$. In addition, we assume that effects of interspecific competition are negligible, i.e., 
$m_{i j}=0, i \neq j$. This results in the following Lotka-Volterra type model

$$
\begin{cases}x^{\prime}(t)=r x(1-x / K)-\sum_{i=1}^{n} p_{i} y_{i} x, & x(0)>0, \\ y_{i}^{\prime}(t)=y_{i}\left(c_{i} p_{i} x-d_{i}-m_{i} y_{i}\right), & y_{i}(0)>0,\end{cases}
$$

$i=1,2, \ldots, n$. If a positive steady state exists, then it must be unique and

$$
x^{*}=\frac{r K+K \sum_{i=1}^{n} p_{i} d_{i} m_{i}^{-1}}{r+K \sum_{i=1}^{n} p_{i}^{2} c_{i} m_{i}^{-1}} .
$$

In order to have $y_{i}^{*}>0, i=1, \ldots, n$, it is necessary that

$$
x^{*}>\frac{d_{i}}{c_{i} p_{i}}=x_{i}^{b}, \quad i=1,2, \ldots, n .
$$

Inequality (3.3) is equivalent to the assumption that at this potential positive steady state, the prey biomass should be above the break-even level for all the predators $y_{i}, i=1,2, \ldots, n$.

We would like to point out that the assumption that the effects of interspecific competition are negligible is commonly assumed in the literature (Armstrong and McGehee, 1980; Li and Smith, 2002). This may happen when species form tight groups or colonies, such as bees and ants. Nevertheless, this constitutes a highly idealized assumption, as most species will inevitably endure some levels of interspecific competition.

For the convenience of studying the global dynamics of model (3.1) when it has no positive steady state, we number the predators in a strict ascending order according to their break-even prey biomasses, that is

$$
x_{i}^{b}<x_{i+1}^{b}, \quad i=1,2, \ldots, n-1 .
$$

Let

$$
x_{j}^{*}=\frac{r K+K \sum_{i=1}^{j} p_{i} d_{i} m_{i}^{-1}}{r+K \sum_{i=1}^{j} p_{i}^{2} c_{i} m_{i}^{-1}} .
$$

Since $x_{i}^{b}<K$, we see that $K>x_{1}^{*}>x_{1}^{b}$. If for some $i \in\{1,2, \ldots, n-1\}, x_{i}^{b} \leq$ $x_{i}^{*}<x_{i+1}^{b}$ then it is easy to show that $x_{j+1}^{*}<x_{j+1}^{b}$ for $j \geq i, j \in\{1,2, \ldots, n-1\}$. Let

$$
x_{n+1}=K \text {. }
$$

Then there is a unique positive integer $l \in\{1,2, \ldots, n\}$ such that

$$
x_{l}^{b} \leq x_{l}^{*}<x_{l+1}^{b} .
$$




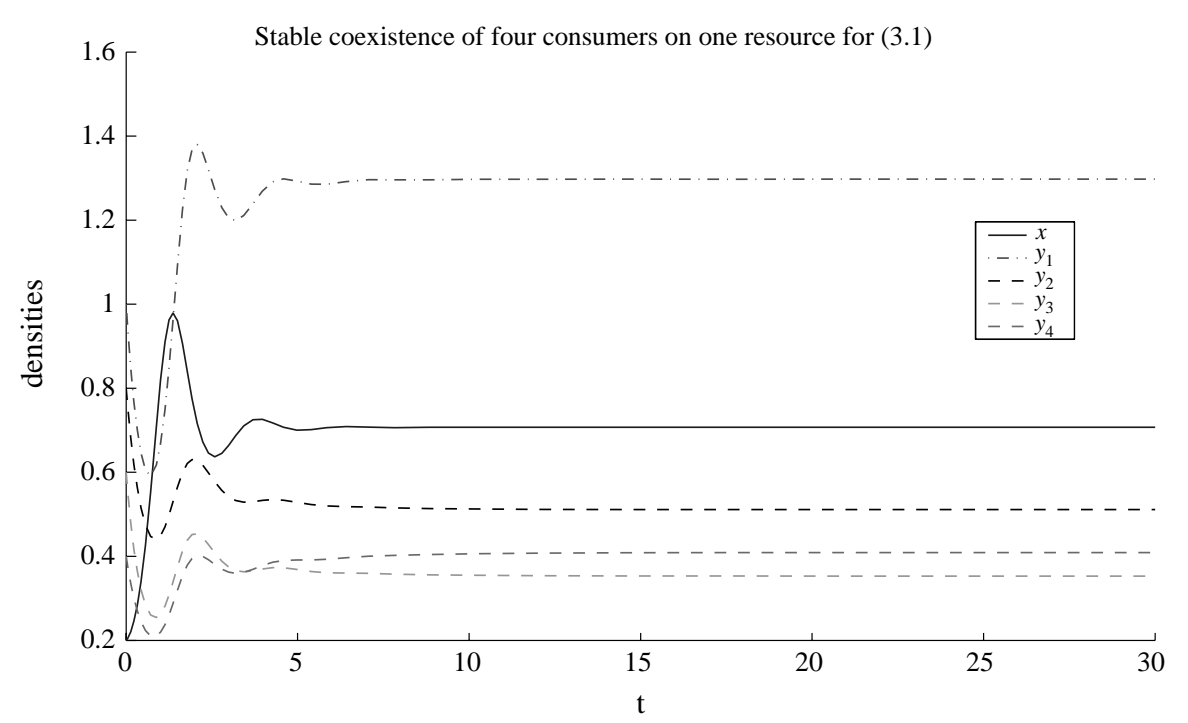

Figure 3. A solution of (3.1) with $n=4, r=5, K=2.5, p_{1}=1, p_{2}=2, p_{3}=$ $1.5, p_{4}=1.8, d_{1}=2, d_{2}=1, d_{3}=1.8, d_{4}=1.5, m_{1}=0.71, m_{2}=0.81, m_{3}=$ $0.91, m_{4}=1, c_{1}=3, c_{2}=1, c_{3}=2, c_{4}=1.5$ and initial condition $(0.2,1,0.8,0.6,0.4)$. This solution tends to the globally stable positive steady state $E^{*}$.

Figure 3 illustrates a solution of model (3.1) with $n=4$ which clearly tends to a globally stable positive steady state $E^{*}$.

It is well known that all positive solutions of model (3.1) tend to the positive steady state (if it exists) or some unique boundary steady state (Case and Casten, 1979). However, it is not known which of the numerous boundary steady states (about $2^{n}$ of them) may serve as the global attractor. The following theorem answers this question and provides an almost complete description of the global qualitative dynamics of model (3.1). The only exceptions are the more tedious degenerate cases that occur with zero probability.

Theorem 3.1. Assume that in (3.1), $x_{i}^{b}<x_{i+1}^{b}, i=1,2, \ldots, n-1$ and $x_{l}^{b}<x_{l}^{*}<x_{l+1}^{b}, l \in\{1,2, \ldots, n\}$. Let

$$
x^{*}=x_{l}^{*}, \quad y_{i}^{*}=\max \left\{0,\left(c_{i} p_{i} x_{l}^{*}-d_{i}\right) / m_{i}\right\} .
$$

Then the nonnegative steady state $E^{*}=\left(x^{*}, y_{1}^{*}, \ldots, y_{n}^{*}\right)$ of system (3.1) is globally attractive (i.e., all positive solutions tend to $E^{*}$ ).

Theorem 3.1 implies that a necessary and sufficient condition for the existence of a positive steady state $E^{*}$ in (3.1) is $x^{*}>x_{M}\left(=x_{n}^{b}\right)$. Theorem 3.1 states that solutions of (3.1) tend to a single nonnegative steady state $E^{*}=\left(x^{*}, y^{*}\right)$. Hence, no paradox of enrichment (Rosenzweig, 1971) is possible for (3.1). For convenience, we call the prey component value at steady state $E^{*}$ the system-wide prey equilibrium biomass of (3.1). Consumer $y_{i}$ persists if and only if its break-even 
prey biomass is less than the system-wide prey equilibrium biomass of (3.1). The following are some important and direct biological implications of Theorem 3.1.

1. Increasing patch size or quality (mediated by the carrying capacity $K$ ) and/or resource growth rate will increase $x_{j}^{*}, j=1, \ldots, n$ and hence $x^{*}$, which in turn increases the likelihood that a given consumer species may persist on the resource.

2. If an invading predator has a break-even prey biomass greater than the system prey equilibrium biomass of (3.1), then the invasion will fail. On the other hand, if its break-even prey biomass is less than the system-wide prey equilibrium biomass of (3.1), then it will successfully invade. Theorem 3.1 confirms the conventional notion that the predator's break-even prey biomass is a key measurement of its competitiveness. If predator A has a lower breakeven prey biomass then predator $\mathrm{B}$, then predator $\mathrm{A}$ is more competitive in the sense that if predator B persists, so will be predator A. However, the CEP no longer holds because predator A can coexist with predator B if the resource has a larger carrying capacity.

3. A successful invasion may cause the extinction of some resident predator species. This would occur if the invasion lowers the system-wide prey equilibrium biomass for (3.1). This can render the reduced system-wide prey equilibrium biomass lower than some resident predators' break even prey biomasses and hence drive them to extinction.

4. Once the system-wide prey equilibrium biomass $x^{*}$ is known, we can use it to estimate the growth rate (doubling time) or extinction rate (half-life) of a consumer species. For example, assume that for consumer species $y$, its break-even prey biomass $x^{b}$ satisfies $x^{b}>x^{*}$. Assume further that all the populations are near the steady-state levels. Then $y^{\prime}(t)=c\left(p\left(x^{*}\right)-p\left(x^{b}\right)\right) y$ which implies that $y(t)=y(0) \exp -c\left(p\left(x^{b}\right)-p\left(x^{*}\right)\right) t$. This gives a halflife of

$$
T=\frac{\ln 2}{c\left(p\left(x^{b}\right)-p\left(x^{*}\right)\right)} .
$$

Figure 4 illustrates some of the implications of Theorem 3.1.

One less obvious but rather important and interesting observation from the proof of Theorem 3.1 is that Theorem 3.1 applies even if some of the consumers lack a direct self-interference mechanism. If such predators exist, then there is one, say $y$, with the smallest break-even prey biomass $x^{b}$. It can be shown that all those self-interfering predators with break-even prey biomasses greater than $x^{b}$ will be eliminated by predator $y$. However, given a sufficiently large resource carrying capacity and/or a sufficiently high resource growth rate, predators with self-interference mechanisms, but lower break-even prey biomasses, may coexist with predator $y$. That is, there is a trade-off between the self-interference and break-even prey biomass that allows coexistence. Moreover, whenever $y$ persists, 
(a) Some isoclines for $\mathrm{r}=2, \mathrm{~K}=5$

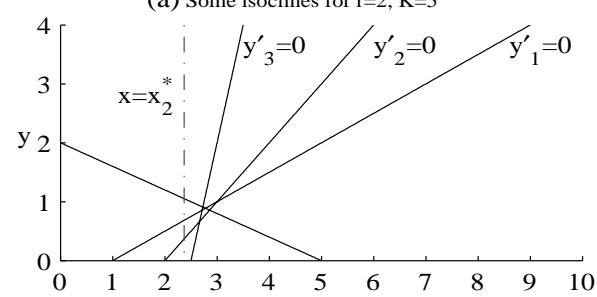

(c) Some isoclines for $\mathrm{r}=2, \mathrm{~K}=10$

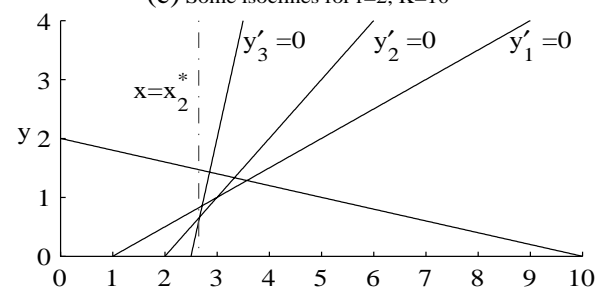

(e) Some isoclines for $\mathrm{r}=4, \mathrm{~K}=5$

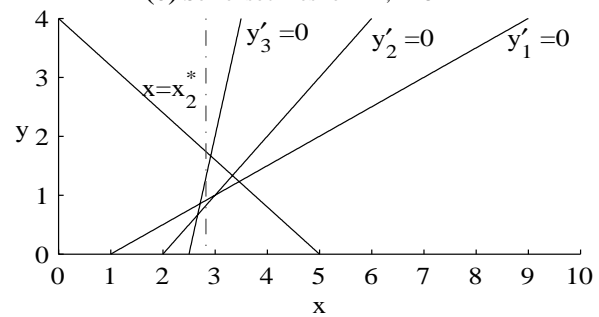

(b) Two predators persist on one prey

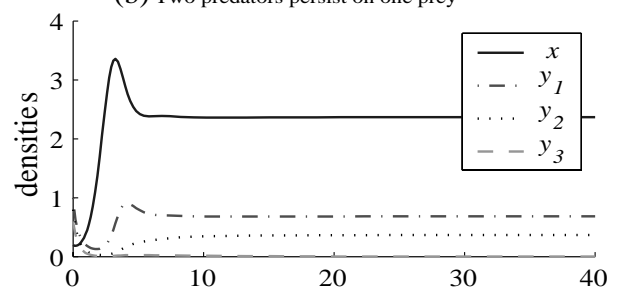

(d) Three predators persist on one prey for $\mathrm{K}=10$

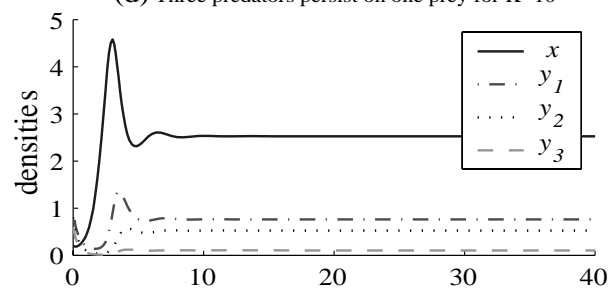

(f) Three predators persist on one prey for $r=4$

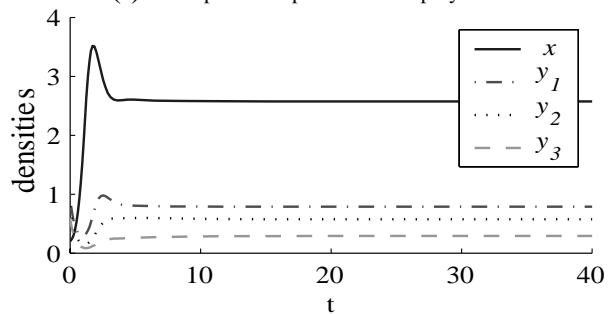

Figure 4. In model (3.1), we assume that $r=2, K=5, p_{1}=1, p_{2}=1, p_{3}=2, d_{1}=$ $1, d_{2}=2, d_{3}=2.5, m_{1}=2, m_{2}=1, m_{3}=0.25, c_{1}=1, c_{2}=1, c_{3}=0.5$ and an initial condition $\left(x(0), y_{1}(0), y_{2}(0), y_{3}(0)\right)=(0.2,1,0.8,0.6)$. The dotted line is $x=x_{2}^{*}$. For this set of parameters, we have $x_{1}^{b}=1, x_{2}^{b}=2, x_{3}^{b}=2.5$. Since $x_{3}^{b}>x_{2}^{*}$ (a), by Theorem 3.1, predator $y_{3}$ dies out (b). When we double the patch size to $K=10$, we then have $x_{3}^{b}<x_{2}^{*}(\mathrm{c})$, and as a result predator $y_{3}$ persists (d). Alternatively, if we double the resource growth rate to $r=4$, we also have $x_{3}^{b}<x_{2}^{*}$ (e), and hence predator $y_{3}$ persists (f).

its break-even prey biomass $x^{b}$ defines the system-wide prey equilibrium biomass $x^{*}$ (i.e., $x^{b}=x^{*}$ ). A simple example of this is illustrated by Fig. 5 .

In the extreme case of identical predator species, we have

$$
d=d_{i}, \quad m=m_{i}, \quad c=c_{i}, \quad p=p_{i}, \quad i=1,2, \ldots, n .
$$

In which case, $x_{i}^{b}=d /(c p) \equiv x_{0}$ and

$$
x^{*}=\frac{r K+K n p d m^{-1}}{r+K n p^{2} c m^{-1}}=\frac{m K r+x_{0} K n p^{2} c}{m r+K n p^{2} c} .
$$

So, $x^{*}>x_{0}=d /(c p)$ if and only if $K>x_{0}$. This will yield a globally attractive positive steady state. This leads us to suggest that when direct predator self-interference exists, predator species are likely to coexist on a single limited resource even if they are very similar. This contrasts with, but does not contradict, previous 

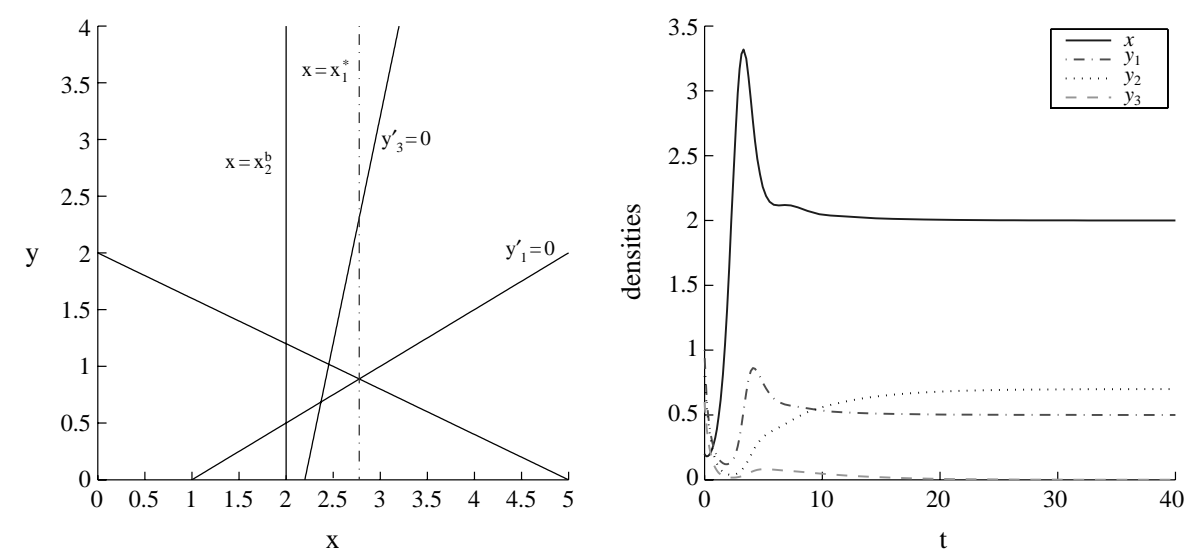

Figure 5. In model (3.1), we assume that $r=2, K=5, p_{1}=1, p_{2}=1, p_{3}=2, d_{1}=$ $1, d_{2}=2, d_{3}=2.2, m_{1}=2, m_{2}=0, m_{3}=0.25, c_{1}=1, c_{2}=1, c_{3}=0.5$ and an initial condition $\left(x(0), y_{1}(0), y_{2}(0), y_{3}(0)\right)=(0.2,1,0.8,0.6)$. The dotted line is $x=x_{1}^{*}$. For this set of parameters, we have $x_{1}^{b}=1, x_{2}^{b}=2, x_{3}^{b}=2.2$. Predator $y_{2}$ eliminates predator $y_{3}$ because $y_{2}$ has a lower break-even prey biomass and does not experience self-limitation. However, $y_{2}$ can coexist with $y_{1}$ because of the trade-off between break-even prey biomass and self-interference. Note that in this case, the system-wide prey equilibrium biomass $x^{*}$ is simply $x_{2}^{b}$.

findings [Abrams (1983), May (2001), based on models without intraspecific consumer interference] that for consumer species to coexist on a single resource, they must be sufficiently different. The absence of contradiction stems from the fact that our model explicitly assumed that the only predator interference is intraspecific, an assumption that should fail to hold if consumer species are too similar. Therefore, direct self-interference provides a simple mechanism for similar species to coexist without resorting to external factors such as spatial and temporal heterogeneities (Loreau and DeAngelis, 1997).

Let

$$
Q=\max \left\{p_{i}^{2} c_{i} / m_{i} \mid i=1,2, \ldots, n\right\}, \quad q=\min \left\{p_{i}^{2} c_{i} / m_{i} \mid i=1,2, \ldots, n\right\} .
$$

Recall that a sufficient condition for the existence of positive steady state $E^{*}$ is $x^{*}>x_{M}$. Since

$$
x^{*} \geq \frac{K r+n K q x_{m}}{r+n K Q},
$$

we see that $x^{*}>x_{M}$ if

$$
r\left(1-\frac{x_{M}}{K}\right)>n\left(Q x_{M}-q x_{m}\right) n \Longleftrightarrow<\frac{r\left(1-x_{M} / K\right)}{Q x_{M}-q x_{m}} .
$$

In order to have the above inequality hold, it is desirable to have large values of $r, K$ and $m_{i}, i=1,2, \ldots, n$, and small values of $n, P-p$ and $x_{M}-x_{m}$. 
Although this confirms the conventional notion and field observations that patch size is positively correlated to biodiversity, we see that the resource growth rate is almost proportional to the maximum number of species that a given resource can support. This suggests that resource growth rates also play key roles in promoting coexistence of consumer species. Simulation work indicates that this property is preserved in more general models (Fig. 4). This finding complements the statement made by Lehman and Tilman (2000) that greater diversity increases community productivity. Observe that if the direct interference strength constants $m_{i}$ are small, then (3.7) fails. This supports the notion that direct interference is vital for the coexistence of some consumers.

\section{Discussion}

In this paper, we made an attempt toward modeling and analyzing simple communities of several predators competing for a single prey. The novel aspect of our model is the incorporation of direct intraspecific competition at the predator trophic level, that is, predator self-interference. Although such interference may or may not be present when predator densities are low (Rosenzweig et al., 1997), it is prevalent when the densities are high. This direct self-interference appears to provide a simple and powerful mechanism that enables the stable coexistence of many predators on a single homogeneous resource. Specifically, we show that in theory, a single resource can sustain simultaneously an unlimited number of different predator species if all these species have a strong self-interference mechanism and satisfy some other generic conditions.

How do our results relate to the CEP? In particular, does the interference among and within species provide additional 'limiting factors' defined in Levin (1970)? The answer is not always confirmative. For example, in model (2.1) the number of 'limiting factors' is $n+1$ with or without interference (i.e., whether $m_{i} j$ or $m_{i}$ are zero or not). The reason is that Levin's argument requires the per-capita growth rates of species to be linear (or to be of special type) functions of limiting factors. In model (2.1), nonlinear functional responses of the consumers, $p_{i}(x)$, already equate the number of 'limiting factors' with the number species. On the other hand, model (3.1) has linear functional responses of the consumers. Without the selfinterference, the minimum number of limiting factors is 2 . The self-interference of each consumer may indeed add a new 'limiting factor'. With all consumers exhibiting self-interference, model (3.1) has $n+1$ 'limiting factors'.

We would like to stress that Theorem 2.1 allows nonlinear self-interference, and moreover, we will comment below that consumers with and without self-interference may coexist simultaneously for the linear case as assumed in Theorem 3.1.

Our most interesting results are Theorem 3.1 and its implications. Although Theorem 3.1 is established for model (3.1), most of its important biological implications should remain true for model (2.1) for the most popular choices of 

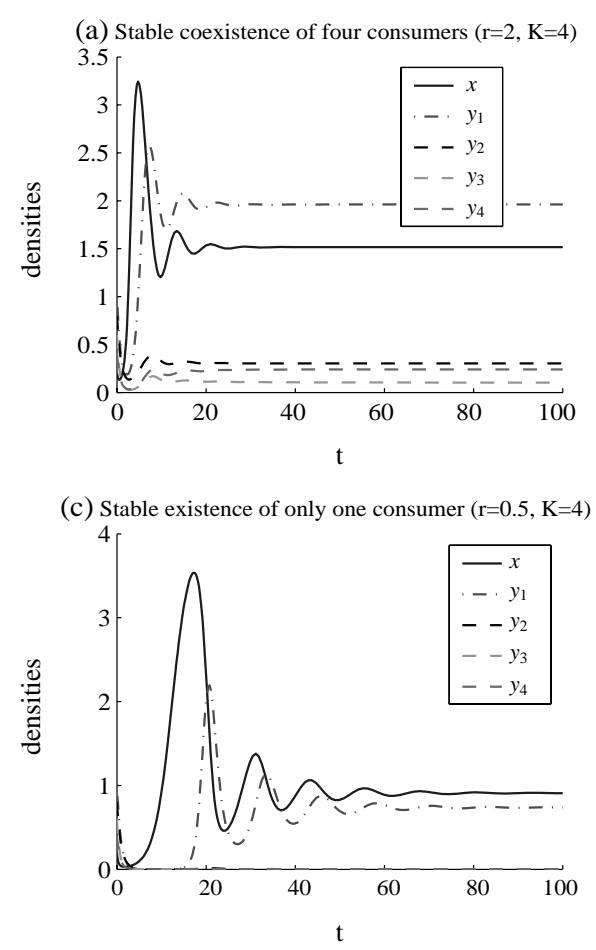

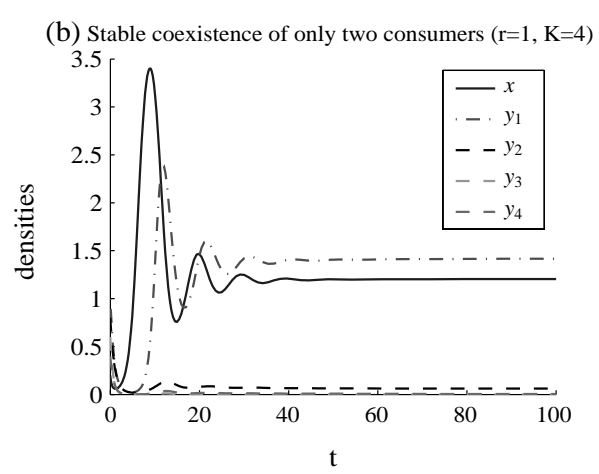

(d) Oscillatory coexistence of four consumers $(\mathrm{r}=2, \mathrm{~K}=8)$

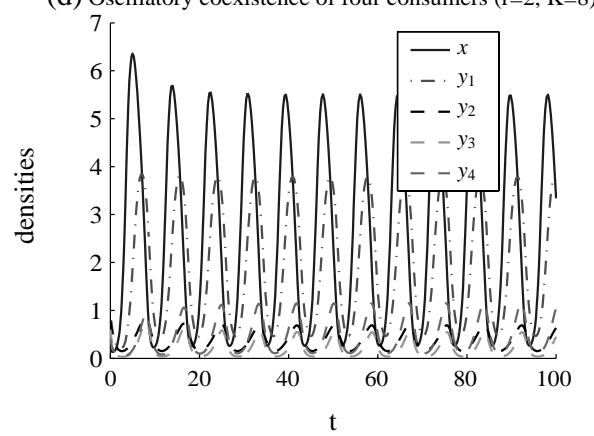

Figure 6. Solutions of (2.1) with $g(x)=1-x / K$ and $p_{i}(x)=p_{i} x /\left(1+a_{i} x\right)$, $i=1,2, \ldots, n$. Here $n=4, p_{1}=1, p_{2}=2, p_{3}=1.5, p_{4}=1.8, d_{1}=1.2, d_{2}=$ $1, d_{3}=1.8, d_{4}=1.5, m_{1}=0.31, m_{2}=0.41, m_{3}=0.51, m_{4}=0.61, c_{1}=3, c_{2}=$ $1, c_{3}=2, c_{4}=1.5, a_{1}=1.0, a_{2}=1.12, a_{3}=0.96, a_{4}=0.98, r$ and $K$ varies, and initial condition $(0.2,1,0.8,0.6,0.4)$. This figure shows that (1) as the resource growth rates decreases, the number of coexistence consumers also declines; (2) when the carrying capacity increases, the solution becomes more oscillatory.

functions and parameters. Figure 6 illustrates this vividly. The model used in Fig. 6 is a Bazykin type model with four predators competing for a single prey. When the prey growth rate is halved, the number of coexisting predators is halved as well. Further halving the prey growth rate removes one additional predator so that only one predator remains. However, for this model, the paradox of enrichment holds true. Doubling the resource's carrying capacity in this particular example induces oscillations in all populations, a result similar to what occurs for the classical Holling type II predator-prey model.

For model (3.1), if interspecific competition occurs among some of the competing species, then as long as the intraspecific competition of each species is stronger than the combined interspecific competition originating from other species, then by the proof of Theorem 3.1 and the essence of the proof of Theorem 2.1 [see also Case and Casten (1979)], the system will continue to have a globally attractive steady state (some species may be extinct at this steady state). However, in 
such cases, it is difficult to identify the losers (facing extinction) and the order of winners as Theorem 3.1 managed to do for the special case when no interspecific competition occurs for any consumer species. Mathematically, the nature of global attractiveness of the steady state identified by Theorem 3.1 allows us to say that when interspecific competition is very small in comparison to intraspecific competition, then Theorem 3.1 can be used as an approximation to provide some useful guidance in identifying consumer species in danger of extinction.

Recent work of Rosenzweig $(1999,2001)$ suggests that the number of species sustainable by a given ecosystem is approximately proportional to its area. While the simple formula (3.7) does not support such a statement, (3.7) suggests that doubling the productivity of the resource may double the number of possible coexisting predators. Conversely, if we shrink the carrying capacity, or reduce the resource's growth rate, we may suffer the loss of some predator species, with those consumers lacking strong self-interference mechanisms suffering the most.

As with most work involving mathematical models, our results inevitably have limitations and warrant more broad and in-depth study. First, our model deals with only one prey and the theorems are unlikely to hold in their current forms if more than one prey is present. Second, the direct intraspecific interference, if it exists, may not necessarily be linear as we postulated in our models (i.e., the $m_{i} y_{i}$ term). With suitable adjustments, we expect the key elements of these findings to remain true for models dealing with multiple prey and multiple predator species together with nonlinear intraspecific interference. In general, it is very difficult to distinguish direct self-competition or self-interference in predators from indirectly mediated interference in nature. Thus, strictly speaking, the models discussed here provide only theoretical alternatives to the many models that treat the direct or indirect intraspecific competition differently. It is fair to say however that this simple treatment of direct intraspecific competition captures the essence of observed negative correlations between conspecific population density and consumer per-capita growth rate. Most importantly, it allows us to examine the strong linkages among notions of biodiversity, resource growth rates and habitat area.

\section{ACKnowledgements}

We would like to thank Peter Abrams, Michael Rosenzweig and Lev Ginzburg for providing us with many important references. This manuscript benefited from many invaluable e-mail exchanges with Peter Abrams and discussions with Chris Klausmeier, Michael Rosenzweig, Hal Smith, Claudia Neuhauser and Simon Levin. Last but not least, we are grateful to the two referees for their accurate and insightful comments that improved this manuscript. The first author's work is partially supported by NSF grant DMS-0077790. I L has been partially supported by NSF grant DEB-0083566 and the Andrew W Mellon Foundation. 


\section{APPENDIX}

Proof of Theorem 2.1. We assume that (2.1) has the positive steady state $E^{*}=\left(x^{*}, y^{*}\right)$ and $\alpha>0$. Let $p_{i}^{*}=p_{i}^{\prime}\left(x^{*}\right)$. Then the variational system of (2.1) at $E^{*}$ is

$$
\left\{\begin{array}{l}
x^{\prime}(t)=-\alpha\left(x-x^{*}\right)-\sum_{i=1}^{n} p_{i}\left(x^{*}\right)\left(y_{i}-y_{i}^{*}\right), \\
y_{i}^{\prime}(t)=y_{i}^{*} c_{i} p_{i}^{*}\left(x-x^{*}\right)-m_{i} y_{i}^{*}\left(y_{i}-y_{i}^{*}\right)-\sum_{j \neq i}^{n} m_{i j} y_{i}^{*}\left(y_{j}-y_{j}^{*}\right) .
\end{array}\right.
$$

Consider

$$
V(x, y)=\left(x-x^{*}\right)^{2}+\sum_{i=1}^{n}\left(c_{i} p_{i}^{*} y_{i}^{*}\right)^{-1} p_{i}\left(x^{*}\right)\left(y_{i}-y_{i}^{*}\right)^{2} .
$$

Then its derivative along any solution of (A.1) is

$$
\begin{aligned}
\left.\Delta \equiv \frac{d V}{d t}(x(t), y(t))\right|_{(\mathrm{A} .1)}= & -2\left[\alpha\left(x-x^{*}\right)^{2}+\sum_{i=1}^{n} \frac{m_{i} p_{i}\left(x^{*}\right)}{c_{i} p_{i}^{*}}\left(y_{i}-y_{i}^{*}\right)^{2}\right. \\
& \left.+\sum_{i=1}^{n} \frac{p_{i}\left(x^{*}\right)}{c_{i} p_{i}^{*}}\left(y_{i}-y_{i}^{*}\right) \sum_{j \neq i}^{n} m_{i j}\left(y_{j}-y_{j}^{*}\right)\right] .
\end{aligned}
$$

Using the simple fact that $2\left(y_{i}-y_{i}^{*}\right)\left(y_{j}-y_{j}^{*}\right) \leq\left(y_{i}-y_{i}^{*}\right)^{2}+\left(y_{j}-y_{j}^{*}\right)^{2}$, we have

$$
\begin{aligned}
\Delta \leq-\left[2 \alpha\left(x-x^{*}\right)^{2}\right. & +\sum_{i=1}^{n}\left(\frac{p_{i}\left(x^{*}\right)}{c_{i} p_{i}^{*}}\left(2 m_{i}-\sum_{j \neq i}^{n} m_{i j}\right)\right. \\
& \left.\left.-\sum_{j \neq i}^{n} \frac{m_{j i} p_{j}\left(x^{*}\right)}{c_{j} p_{j}^{*}}\right)\left(y_{i}-y_{i}^{*}\right)^{2}\right] \leq 0 .
\end{aligned}
$$

Clearly $\left.\frac{d V}{d t}(x(t), y(t))\right|_{(\mathrm{A} .1)}=0$ if and only if $(x(t), y(t))=E^{*}$. This shows that $V(x, y)$ is a Lyapunov function (Hale, 1980) for (A.1) and the theorem follows.

Proof of Theorem 3.1. Observe first that $E^{*}=\left(x^{*}, y_{1}^{*}, \ldots, y_{n}^{*}\right)$ is indeed a nonnegative steady state of (3.1) with $y_{i}^{*}>0$ for $1 \leq i \leq l$ and $y_{i}^{*}=0$ for $i>l$. Then

$$
\left\{\begin{aligned}
& x^{\prime}(t)=x {\left[-(r / K)\left(x-x^{*}\right)-\sum_{i=1}^{l} p_{i}\left(y_{i}-y_{i}^{*}\right)-\sum_{i=l+1}^{n} p_{i}\left(y_{i}-y_{i}^{*}\right)\right], } \\
& x(0)>0, y_{i}\left[c_{i} p_{i}\left(x-x^{*}\right)-m_{i}\left(y_{i}-y_{i}^{*}\right)-\delta_{i}\right], \\
& y_{i}(0)>0,
\end{aligned}\right.
$$


where $\delta_{i}=0$ for $1 \leq i \leq l$ and $\delta_{i}=-c_{i} p_{i}\left(x^{*}-x_{i}^{b}\right)>0$ for $i>l$. Consider

$$
V(x, y)=x-x^{*}-x^{*} \ln x+\sum_{i=1}^{n} c_{i}^{-1}\left(y_{i}-y_{i}^{*}-y_{i}^{*} \ln y_{i}\right) .
$$

Then its derivative along any solution of (3.1) is

$$
\begin{aligned}
\left.\frac{d V}{d t}(x(t), y(t))\right|_{(3.1)} & =-\left[\frac{r}{K}\left(x-x^{*}\right)^{2}+\sum_{i=1}^{n} c_{i}^{-1} m_{i}\left(y_{i}-y_{i}^{*}\right)^{2}+\sum_{i=l+1}^{n} c_{i} \delta_{i} y_{i}\right] \\
& \leq 0
\end{aligned}
$$

Clearly $\left.\frac{d V}{d t}(x(t), y(t))\right|_{(3.1)}=0$ if and only if $(x(t), y(t))=E^{*}$. This shows that $V(x, y)$ is a Lyapunov function for (3.1) and the conclusion of the theorem follows.

\section{REFERENCES}

Abrams, P. A. (1980). Consumer functional response and competition in consumerresource systems. Theor. Popul. Biol. 17, 80-102.

Abrams, P. A. (1983). The theory of limiting similarity. Annu. Rev. Ecol. Syst. 14, 359-376.

Abrams, P. A. (1984a). Variability in resource consumption rates and the coexistence of competing species. Theor. Popul. Biol. 25, 106-245.

Abrams, P. A. (1984b). Foraging time optimization and interactions in food webs. Am. Nat. 124, 80-96.

Abrams, P. A. (1986). Character displacement and niche shift analyzed using consumerresource models of competition. Theor. Popul. Biol. 29, 107-160.

Abrams, P. A. (1987). Alternative models of character displacement and niche shift. 2. Displacement when there is competition for a single resource. Am. Nat. 130, 271-282.

Abrams, P. A. (1998). High competition with low similarity and low competition with high similarity: exploitative and apparent competition in consumer resource system. Am. Nat. 152, 114-128.

Abrams, P. A. (2001). The effect of density-independent mortality on the coexistence of exploitative competitors for renewing resources. Am. Nat. 158, 459-470.

Armstrong, R. A. and R. McGehee (1976). Coexistence of species competing for shared resources. Theor. Popul. Biol. 9, 317-328.

Armstrong, R. A. and R. McGehee (1980). Competitive exclusion. Am. Nat. 115, 151-170.

Bazykin, A. D., A. I. Khibnik and B. Krauskopf (1998). Nonlinear Dynamics of Interacting Populations, Singapore: World Scientific.

Cavani, M. and M. Farkas (1994). Bifurcations in a predator-prey model with memory and diffusion. I. Andronov-Hopf bifurcation. Acta Math. Hungar. 63, 213-229.

Case, T. J. and R. G. Casten (1979). Global stability and multiple domains of attraction in ecological systems. Am. Nat. 113, 705-714.

Case, T. J. and M. E. Gilpin (1974). Interference competition and niche theory. Proc. Natl Acad. Sci. USA 71, 3073-3077.

Collier, T. R. and M. S. Hunter (2001). Lethal interference competition in the whitefly parasitoids Eretmocerus eremicus and Encarsia sophia. Oecologia 129, 147-154. 
Cosner, C., D. L. DeAngelis, J. S. Ault and D. B. Olson (1999). Effects of spatial grouping on the functional response of predators. Theor. Popul. Biol. 56, 65-75.

DeAngelis, D. L., R. A. Goldstein and R. V. O'Neill (1975). A model for trophic interaction. Ecology 56, 881-892.

Denno, R. F. and G. K. Roderick (1992). Density-related dispersal in plant-hoppers: effects of intraspecific crowding. Ecology 73, 1323-1334.

Durant, S. (1998). Competition refuges and coexistence: an example from Serengeti carnivores. J. Anim. Ecol. 67, 370-386.

Farkas, M. and H. I. Freedman (1989). The stable coexistence of competing species on a renewable resource. J. Math. Anal. Appl. 138, 461-472.

Fox, L. R. (1975). Cannibalism in natural populations. Annu. Rev. Ecol. Syst. 6, 87-106.

Freedman, H. I. (1980). Deterministic Mathematical Models in Population Ecology, New York: Marcel Dekker.

Goh, B. S. (1977). Global stability in many species systems. Am. Nat. 111, 135-143.

Grosholz, E. D. (1992). Interactions of intraspecific, interspecific, and apparent competition with host-pathogen interactions. Ecology 73, 507-514.

Grover, J. P. (1997). Resource Competition, London, UK: Chapman \& Hall.

Hale, J. K. (1980). Ordinary Differential Equations, Malabar, FL: Krieger.

Hardin, G. (1960). Competitive exclusion principle. Science 131, 1292-1297.

Harrison, G. W. (1995). Comparing predator-prey models to Luckinbill's experiment with Didinium and Paramecium. Ecology 76, 357-374.

Hastings, A. (1980). Disturbance, coexistence, history and competition for space. Theor. Popul. Biol. 18, 363-373.

Holt, R. D. and G. A. Polis (1997). A theoretical framework for intraguild predation. Am. Nat. 149, 745-764.

Hsu, S. B., K. S. Cheng and S. P. Hubbell (1981). Exploitative competition of microorganism for two complementary nutrients in continuous culture. SIAM J. Appl. Math. 41, 422-444.

Hwang, T.-W. and Y. Kuang (2002). Rich global qualitative dynamics of Bazykin model (preprint).

Huisman, J. and F. J. Weissing (1999). Biodiversity of plankton by species oscillations and chaos. Nature 402, 407-410.

Huisman, J. and F. J. Weissing (2000). Coexistence and resource competition. Nature 407, 694.

Huisman, J. and F. J. Weissing (2001). Fundamental unpredictability in multispecies competition. Am. Nat. 157, 488-494.

Hutchinson, G. E. (1961). The paradox of the plankton. Am. Nat. 95, 137-145.

Jervis, M. A. and N. A. C. Kidd (1986). Host feeding strategies in hymenopteran parasitoids. Biol. Rev. 61, 395-434.

Karolyi, G., A. Pentek, I. Scheuring, T. Tel and Z. Toroczkai (2000). Chaotic flow: the physics of species coexistence. Proc. Natl Acad. Sci. USA 97, 13661-13665.

Kendall, B. E., J. Prendergast and O. N. Bjornstad (1998). The macroecology of population dynamics: taxonomic and biogeographic patterns in population cycles. Ecol. Lett. 1, 160-164.

Kendall, B. E., C. J. Briggs, W. W. Murdoch, P. Turchin, S. P. Ellner, E. McCauley, R. M. Nisbet and S. N. Wood (1999). Why do populations cycle? A synthesis of statistical and mechanistic modelling approaches. Ecology 80, 1789-1805. 
Kinzig, A. P., S. A. Levin, J. Dushoff and S. Pacala (1999). Limiting similarity, species packing, and system stability for hierarchical competition-colonization models. Am. Nat. 153, 137-145.

Krebs, J. R. and N. B. Davies (1981). An Introduction to Behavioural Ecology, London: Blackwell Scientific Publications.

Krukonis, G. and W. M. Schaffer (1991). Population cycles in mammals and birds: does periodicity scale with body size? J. Theor. Biol. 148, 469-493.

Kuang, Y. (1990). Global stability of Gause-type predator-prey systems. J. Math. Biol. 28, 463-474.

Kuznetsov, Y. A. (1998). Elements of Applied Bifurcation Theory, 2nd edn, Applied Mathematical Sciences 112, New York: Springer.

Lehman, C. L. and D. Tilman (2000). Biodiversity, stability, and productivity in competitive communities. Am. Nat. 156, 534-552.

Levin, S. A. (1970). Community equilibria and stability, and an extension of the competitive exclusion principle. Am. Nat. 104, 413-423.

Levin, S. A. (1999). Fragile Dominion: Complexity and the Commons, Reading, MA: Perseus Books.

Li, B. and H. Smith (2002). How many species can two essential resources support? SIAM J. Appl. Math. 62, 336-366.

Loladze, I., Y. Kuang and J. J. Elser (2000). Stoichiometry in producer-grazer systems: linking energy flow with element cycling. Bull. Math. Biol. 62, 1137-1162.

Loladze, I., Y. Kuang, J. Elser and W. Fagan (2003). Coexistence of two predators on one prey mediated by stoichiometry. Theor. Popul. Biol. (at press).

Loreau, M. and D. L. DeAngelis (1997). Source-sink dynamics and the coexistence of species on a single resource. Theor. Popul. Biol. 51, 79-93.

MacArthur, R. and R. Levins (1964). Competition, habitat selection, and character displacement in a patchy environment. Proc. Natl Acad. Sci. USA 51, 1207-1210.

May, R. M. (2001). Stability and Complexity in Model Ecosystems (Princeton Landmarks in Biology), Princeton: Princeton University Press.

Myers, R. A., N. J. Barrowman, J. A. Hutchings and A. A. Rosenberg (1995). Population dynamics of exploited fish stocks at low population levels. Science 269, 1106-1108.

McCann, K., A. Hastings and G. R. Huxel (1998). Weak trophic interactions and the balance of nature. Nature 395, 794-798.

McCann, K. S. (2000). The diversity-stability debate. Nature 405, 228-233.

Neuhauser, C. (1998). Habitat destruction and competitive coexistence in spatially explicit models with local interactions. J. Theor. Biol. 193, 445-463.

Rosenzweig, M. L. and R. H. MacArthur (1963). Graphical representation and stability conditions of predator-prey interactions. Am. Nat. 97, 209-223.

Rosenzweig, M. L. (1971). Paradox of enrichment: destabilization of exploitation systems in ecological time. Science 171, 385-387.

Rosenzweig, M. L. (1999). Heeding the warning in biodiversity's basic law. Science 284, 276-277.

Rosenzweig, M. L. (2001). The four questions: what does the introduction of exotic species do to diversity? Evol. Ecol. Res. 3, 361-367.

Rosenzweig, M. L., Z. Abramsky and A. Subach (1997). Safety in numbers; sophisticated vigilance by Allenby's gerbil. Proc. Natl Acad. Sci. USA 94, 5713-5715.

Ruan, S. and X.-Z. He (1998). Global stability in chemostat-type competition models with nutrient recycling. SIAM J. Appl. Math. 58, 170-192. 
Skalski, G. T. and J. F. Gilliam (2001). Functional responses with predator interference: viable alternatives to the Holling type II model. Ecology 82, 3083-3092.

Strobeck, C. (1973). N species competition. Ecology 54, 650-654.

Tanner, J. T. (1966). Effects of population density on growth rates of animal populations. Ecology 47, 733-745.

Tannerfeldt, M., B. Elmhagen and A. Angerbjorn (2002). Exclusion by interference competition? The relationship between red and arctic foxes. Oecologia 132, 213-220.

Tilman, D. (1994). Competition and biodiversity in spatially structured habitats. Ecology 75, 2-16.

Vance, R. R. (1984). Interference competition and the coexistence of two competitors on a single limiting resource. Ecology 65, 1349-1357.

Vance, R. R. (1985). The stable coexistence of two competitors for one resource. Am. Nat. 126, 72-86.

Volterra, V. (1926). Fluctuations in the abundance of a species considered mathematically. Nature 118, 558-560.

Received 31 May 2002 and accepted 9 January 2003 